\section{Frustrated Liquids: Liquid Crystal Blue Phases}

\section{P. Pieranski, Orsay and P.E. Cladis, Murray Hill}

(Physique des Solides, Université Paris-Sud and AT\&T Bell Laboratories)

\begin{abstract}
Because the locally favourable structure is globally unfavourable, cholesteric liquid crystals are frustrated. Near the isotopic transition, blue phases (BP) appear as compromise structures to relieve this frustration. BPI and BPII are soft, cubic crystals with giant unit cells.
\end{abstract}

Some remarkable developments in the last 20 years in condensed matter physics can be attributed to the attention that has been paid to the state of matter known as liquid crystals or mesophases where the microscopic organization is between that of an isotropic liquid and the usual crystal. Blue phases are probably the first liquid crystal phases to have been observed. The Austrian botanist, Reinitzer noted in 1888 when cooling the liquid phase of chiral (i.e. containing at least one asymmetric carbon atom) and elongated molecules, "a violet and blue colour phenomenon" the blue phases - "which appears, then quickly disappears, leaving the substance cloudy but still liquid" (identified now as the heliocoidal cholesteric phase) ... " which forms oily streaks in the melt" $\left.{ }^{1}\right)$. In contrast to the helicoidal phase that persists over several deg $C$, the blue phases of which there are three typically exist over a range of fractions of a deg C. This explains why it has taken nearly 100 years to realise that the symmetry and physical properties of blue phases are distinct from the usual helicoidal phase, the cholesteric liquid crystal phase ${ }^{2}$ ).

Here, we describe experiments at Orsay and at Bell Laboratories ${ }^{3-5}$ ) that show directly the molecular organiza- tion of blue phases. We also discuss the notion of geometric frustration applied to chiral molecules ${ }^{6}$ ) since it is believed to be the fundamental reason for the diversity of the cholesteric phase.

\section{Definition of the Blue Phase Order Para- meter}

The organization of blue phases I and II probably corresponds best to the naive picture evoked by the term liquid crystal (Fig. 1). On a microscopic scale, the molecules are as mobile as in the isotropic liquid state. In a volume, $v$, that is small but still contains thousands of molecules, the direction along which the sys tem chooses to orient, $\mathbf{n}$, called the director, results in an anisotropy of the dielectric tensor, $\hat{\varepsilon}(r)$. Its anisotropic part, $\varepsilon_{i j}^{a}=\varepsilon i j-{ }_{3}^{1} \operatorname{tr}(\varepsilon) \delta_{i j}$ distinguishes blue phases from the isotropic liquid and can be treated as an order parameter. It turns out that for blue phases I and II, referred to as BPI and BPII, $\varepsilon_{i j}^{a}(r)$ is invariant with respect to the symmetry operator of a crystallographic group, $\mathrm{G}_{\mathrm{s}}$ the crystal symmetry of which gives to the order parameter, $\hat{\varepsilon}^{\mathrm{a}}(\boldsymbol{r})$ a periodicity. It can be expanded in a Fourier series ${ }^{7}$ ):

$$
\hat{\varepsilon}^{\mathrm{a}}(r)=\sum_{q} \hat{\varepsilon}_{q} \exp \left(\mathrm{i}\left(q r+v_{q}\right)\right)+\mathrm{c} . \mathrm{c}
$$

The lattice constant of the Bravais lattice of $G_{s}$ is giant by crystallographic standards - a unit cell is typically
$0.3 \mu \mathrm{m}$ across and contains some $10^{7}$ molecules. Thus, while on a macroscopic scale BPI and BPII resemble the usual crystal state, because of the large size of the unit cells, they can be considered to be special colloidal crystals ${ }^{8}$ ).

There is a third blue phase called BPIII or simply, the fog, that occurs at a higher temperature than BPI and BPII. Since this phase is not periodic, it appears to be the liquid state analogue of BPI and II.

These unusual structures raise several questions.

1. What is the symmetry and the precise form of the tensor field, $\varepsilon_{i \eta}$ ?

2. What are the physical properties that result?

3. Why should such structures occur in the first place?

A direct way of studying the symmetry and structure of crystals is by observing their shapes and the diffraction patterns they create in convergent light. We have developed an experimental set-up where both can be done simultaneously on the same monocrystal and in the following, we describe some of our observations and discuss how our results help answer some of the ques tions raised above.

Determination of Symmetry and Structure of Blue Phases ${ }^{3.4}$ )

With the experimental set-up shown in Fig. 2, we study both the real-space form of blue phase crystals as well as the symmetry of their Bragg reflections. The apparatus comprises a reflecting, optical microscope with a monochromator providing a light source of wavelength $\lambda$ between $3500 \AA$ and $10000 \AA$. Because of the large lattice constants, the wavelength of the Bragg reflections that measure the Fourier components $\hat{\varepsilon}_{q}$ of the order parameter, fall in this range of wavelengths. The temperature is controlled and stabilized to better than $0.01^{\circ} \mathrm{C}$.

Fig. 1 - Structure of Blue Phases: Local orientational order in a small volume V leads to an anisotropy of a tensor property such as the dielec tric tensor, $\hat{\varepsilon}$.
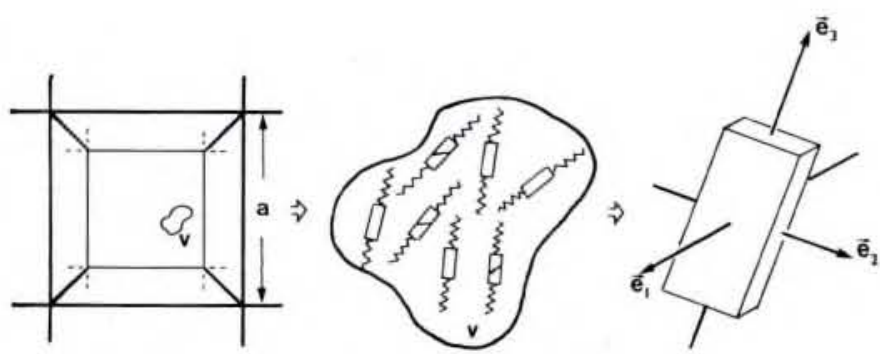

Fig. 2 (Right) - A sample of blue phase, BP, is sandwiched between a glass slide $S$ and a glass cylinder $C$. A monocrystal M grown on $S$ is illuminated by light of wavelength $\lambda$. For $\lambda$ satisfying the Bragg condition for [hkl] planes perpendicular to $O$, the monocrystal appears as a brilliant polyhedron on a dark background. $T_{1}$ and $T_{2}$ are temperatures regulated independently.

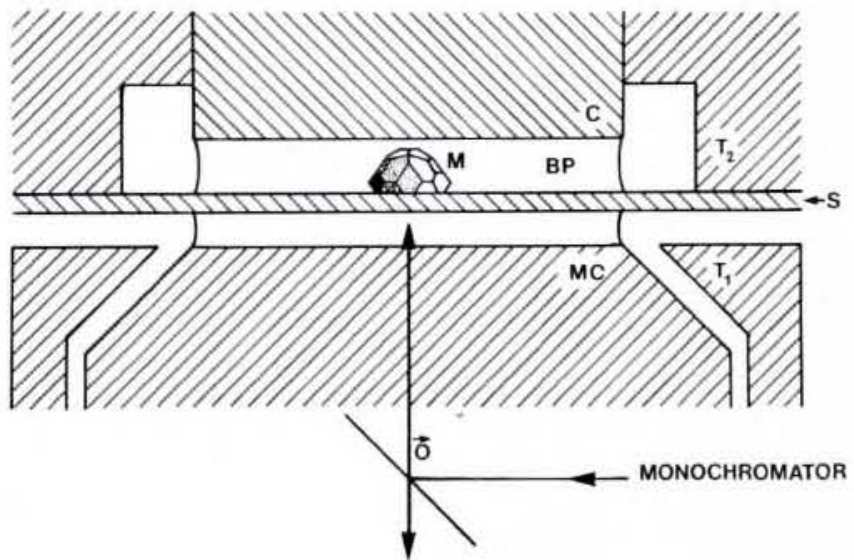



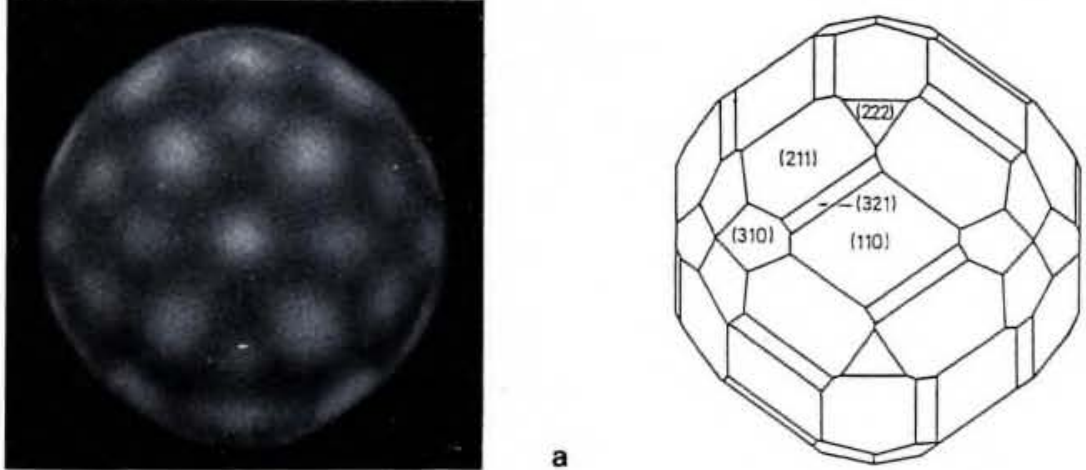

b

Fig. 3 - A large monocrystal of BPI showing the relative disposition of the facets identified in Fig. $3 b$.

\section{Observations in Real Space - Crystal Facets ${ }^{3}$ )}

When the incident wavelength, $\lambda$, satisfies the Bragg reflection condition for a particular Fourier component of the order parameter and the corresponding $q$ is parallel to the microscope axis, the blue phase crystal that nucleated on the colder face of the cell, $\mathrm{S}$ in Fig. 2, and grew in the isotropic liquid phase, appears as a bright polyhedron on a dark background. From such observations, one learns that BPI and BPII have different crystal shapes and Bragg reflections. Only diffuse scattering is associated with BPIII and it shows no facets. Little is known about this phase and we do not discuss it here.

In general, the shape of the crystals is directly related to the symmetry of the lattice. Furthermore, the surfaces are usually composed of facets (smooth) joined by rounded (rough) parts. Facets are a consequence of the translational order or periodicity of the crystal and their relative disposition is determined by point symmetries (rotations and reflections).

The study of blue phase facets is interesting for two reasons. In the first place, empirical methods developed at the turn of the century are available for determining the symmetry of the blue phases.

Fig. 4 a. - Kossel diagrams formed by a monocrystal in a converging light source.

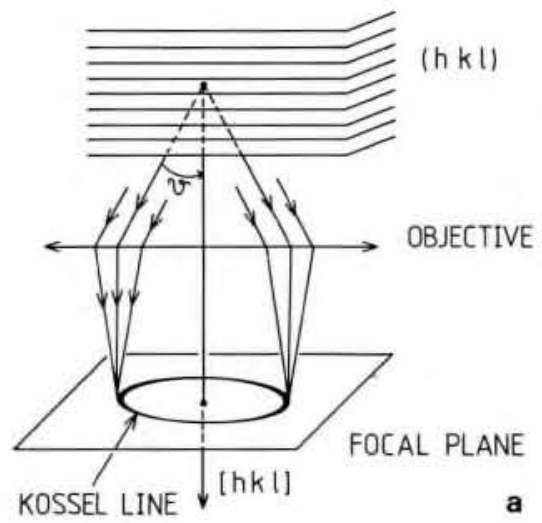

Using these methods, we found that the space group (see insert) of BPI is $\mathrm{O}^{8}(14,32)$ and that of $\mathrm{BPII}$ is $\mathrm{O}^{2}\left(\mathrm{P}_{4}, 32\right)$. Second, there has been a revival of interest recently in the general problem of facetting in crystals. Blue phases constitute a rare system because facets, steps and domain walls can all be directly observed and their dynamics studied in situ.

Fig. 3a shows the arrangement of facets in a large BPI monocrystal. Four kinds of facets can be seen: $\{110\},\{211\}$, $\{310\}$ and $\{222\}$. Fig $3 b$ shows $\{321\}$ facets observed on larger monocrystals. The locations of these implies that there is no centre of symmetry in the crystal morphology and is consistent with the chiral nature of the molecules.

In order of increasing growth rates, the facets are $\{110\},\{211\},\{310\},\{222\}$ and $\{321\}$. According to the empirical law of Bravais, Donnay and Harker, this is the signature of the group $\mathrm{O}^{8}(14,32)$ for which the Bravais lattice is body centred cubic. The helicoidal nature of the fourfold screw axis, $4_{1}$, accounts for the absence of $\{100\}$ facets.

\section{Observations in q-space - Kossel Diagrams ${ }^{4}$ )}

In addition to these real-space observations, Kossel diagrams (Fig. 4) can be

Fig. 4 b. - The Kossel diagram of BPI viewed along the [110] direction. BPI is bcc.

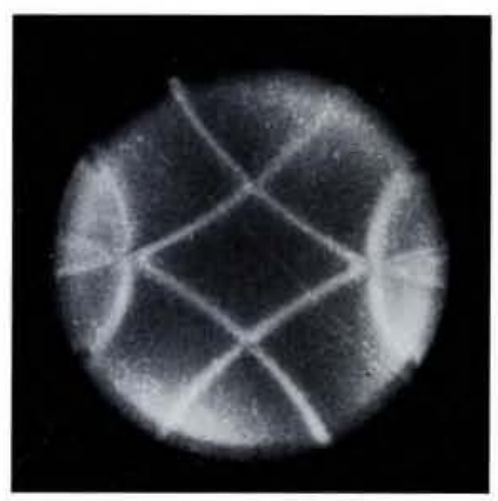

The Bravais lattice corresponding to the space groups $0^{2}$ and $0^{8}$ are respectively simple cubic and body centred cubic. Let $a$ be the dimension of a cubic unit cell. The fourfold axes $4_{1}$ and $4_{2}$ are screw axes; a rotation of $2 \pi / 4$ is followed by translations of $a / 4\left(4_{1}\right)$ and $2 a / 4\left(4_{2}\right)$ along the axes. of Buerger M.J., Elementary crystallography (Wiley) 1956.

observed in the focal plane of the microscope objective. They are diffraction patterns formed by a monocrystal in the focal plane of the microscope objective in a converging beam of light. Since each Kossel ring corresponds to a particular Fourier component, such diagrams are finger-prints of crystal structure.

Fig. 4b shows the Kossel diagram for BPI when the centre of the [110] Kossel ring is in the centre of the photograph. The pattern is formed by arcs of Kossel rings from [101], [10 $\overline{1}],[011]$ and [01 $\overline{1]}$ planes. In addition, bright [200] diffraction rings intersect the crossing point of the [101] and [101] arcs and [020] rings intersect the [011] and [011] arcs. The angular distance between different [hkl] planes as well as the distance, $d_{\text {hkl }}$ between [hkl] planes can be deduced from this observation. The pattern shown in Fig. $4 b$ is the signature of a body centred cubic lattice (bcc) viewed along the [110] direction.

Fig. $4 \mathrm{c}$ shows the Kossel pattern for BPII, characteristic of a simple cubic lattice (sc) observed along the [110] direction. Here the larger pair of diffraction rings come from the [100] and [010] planes while the small circle that intersects their crossing points is from [110] planes.

As the wavelength is changed, the Bragg angles change as a function of the lattice structure and the Kossel patterns change in a way that is consistent with the assignment bcc for BPI and sc for BPII.

Fig. 4 c. - The Kossel diagram of BPII viewed along the [110] direction. BPII is sC.

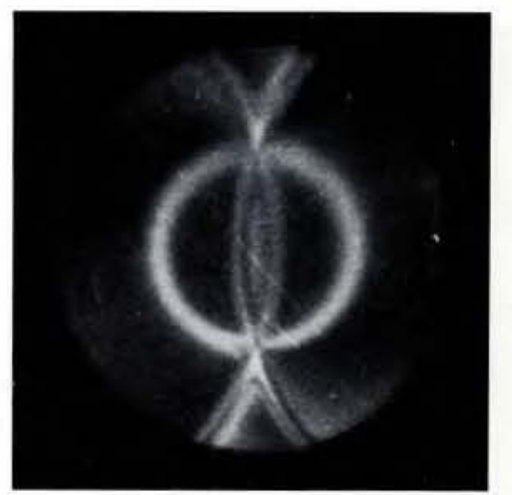


Orientation of Blue Phase Crystals by Electric Fields ${ }^{5}$ )

We find that electric fields orient blue phase crystals so that a 4-fold axis is parallel to the field. We account for this by considering the torque, $\Gamma=P \times E$ exerted on the polarization $\boldsymbol{P}$ by an electric field $E$. Because of the cubic symmetry of blue phases, the second rank susceptibility tensor, $\xi_{i j}$, is isotropic and cannot contribute to the orientation of the crystal. Higher rank susceptibility tensors must be

1) invariant under the operations of the point group $\mathrm{O}(432)$ and

2) symmetric when indices are interchanged and the frequency of the electric fields is the same. There is no third rank tensor satisfying these conditions but there are two fourth rank tensors, $\xi_{\alpha \beta \gamma \delta}$ which do: an isotropic one,

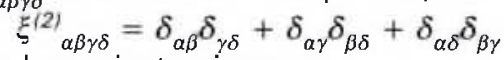
and an anisotropic one

$$
\xi^{(1)}{ }_{\alpha \beta \gamma \delta}=\sum_{i=1}^{3} n_{\alpha}^{i} n_{\beta}^{i} n_{\gamma}^{i} n_{\delta}^{i}
$$

where $n^{1}, n^{2}$ and $n^{3}$ are parallel to the 4-fold axes of the crystal. The total nonlinear susceptibility is a linear combination of these two quantities:

$$
\xi_{\alpha \beta \gamma \delta}=A \xi^{(1)}{ }_{\alpha \beta \gamma \delta}+B \xi^{(2)}{ }_{\alpha \beta \gamma \delta} .
$$

The torque exerted on a crystal of volume $V$, is then,

$$
\Gamma=V A \sum_{i=1}^{3}\left(n^{i} E\right)^{3}\left(n^{i} \times E\right) .
$$

$\Gamma$ vanishes when the field is:

1) parallel to the 3 -fold axis. This orientation is absolutely unstable when $A>0$ and absolutely stable when $A<0$;

2) parallel to a 2 -fold axis with neutral stability or

3) parallel to a 4-fold axis with absolute stability for $A>0$ and absolute instability for $A<0$.

In intermediate positions, non-zero torques act on the crystal to bring it into the nearest stable position where $\Gamma$ is zero.

\section{Origin of Blue Phases: Geometric Frus-} tration of Chiral Molecules

In the usual helicoidal phase, Fig. 5a, chiral molecules tend to align parallel to a single direction, $\boldsymbol{n}$ in a plane of constant $x . n$ rotates through $2 \pi$ around $x$ over a characteristic length, $p$, called the pitch. Call the energy of this configuration zero. Consider now the case where, in an isolated region of space, $\boldsymbol{n}$ turns simultaneously about two axes, $x$ and $y$ over a distance $r$ (Fig. 5b) with a pitch, $p_{2}$. This structure is known as «double twist" ${ }^{9}$ ) and it has the interesting property that its total energy is negative compared to single twist when $r$ is about $p_{2} / 4$. As $r$ increases beyond this value, double twist becomes more energetic compared to the singly twisted cholesteric phase. Thus, the structure that is locally favourable is globally unfavou-

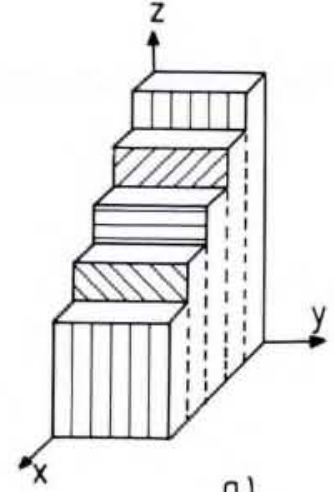

a)

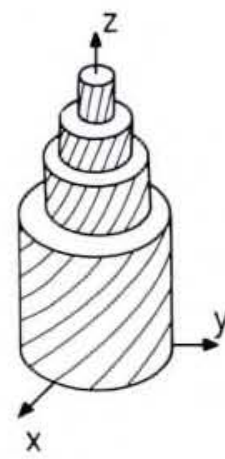

b)

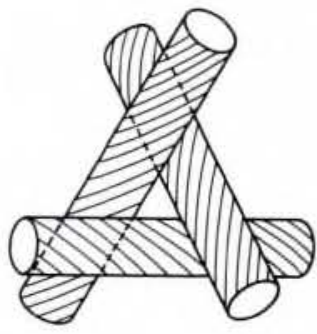

c)
Fig. 5 a) - Schematic of single twist, the structure of the usual helicoidal cholesteric phase. b) - Schematic of double twist the structure that is locally favorable but globally unfavorable compared to single twist. c) - Frustration: A line defect is needed to fill the gap between three mutually orthogonal cylinders of double twist. rable compared to the uniform helicoidal phase.

If three-dimensional Euclidean space is regularly filled with cylinders of double twist ${ }^{9}$ ) but of radius $\cong p_{2} / 4, n$ is in register where the cylinders touch but there are holes in the structure. For example, in the [111] direction, $\boldsymbol{n}$ is in determinate in the gap between three mutually orthogonal adjacent cylinders (Fig. 5c). One way to resolve the conflict is to fill this space with isotropic liquid, but at a cost in energy - the price of compromise - becoming less costly as the isotropic phase is approached. This accounts for the blue phases lying between the isotropic liquid and the helicoidal phase.

Cholesteric blue phases are one example among many of the diverse structures than can arise from geometric frustration. Other examples may be found in the so-called Frank-Kasper phases, amorphous systems, liquid crystal polymers and lyotropic liquid crystals. The notion of frustration thus appears as a fruitful new idea that can be expected to lead to many new insights.

\section{Conclusions}

Although neglected or poorly understood for nearly a century, because of their remarkable structure, their unusual physical properties and the theoretical questions they raise, blue phases deserve the interest they have finally been paid. New ideas generated to account for these phases will further our understanding of other systems of condensed matter physics.

\section{REFERENCES}

1. Quoted from H. Kelker, "History of Liquid Crystals», Mol. Cryst. Liq. Cryst. 21 (1973) 1.

2. For a recent review see for example: Belyakov V.A. and Dmitrienko V.E., Sov. Phys. Uspekii 28 (1985) 535.

3. Barbet-Massin R., Cladis P.E. and Pieranski P., Phys. Rev. A30 (1984) 1161.

Pieranski P., Barbet-Massin R. and Cladis P.E., Phys. Rev. A31 (1985) 3912.

4. Pieranski P. and Cladis P.E., "BPX: A Field Induced Tetragonal Blue Phase" submitted to Phys. Rev. A.

5. Pieranski P., Cladis P.E., Garel T. and Barbet-Massin R., J. de Physique 47(1986) 139.

6. Sethna J.P., Phys. Rev. 31(1985) 6279.

7. Grebel M., Hornreich R.M. and Shtrikman S., Phys. Rev. A28(1983) 1114. Winter Workshop on Colloidal Crystals" eds. P. Pieranski and F. Rothen, J. de Physique 46(1985) 1-326

9. Meiboom S., Sethna J.P., Anderson P.W. and Brinkman W.F., Phys. Rev. Lett. 46(1981) 1216.
8. See for example: «Proceedings of the

\section{UNIVERSITY OF WARWICK Professorship in Experimental Physics}

Applications are invited for a Professorship in Experimental Physics, in succession to Professor A.J. Forty, who has just taken up the post of Principal and Vice-Chancellor of the University of Stirling.

Candidates should have expertise in the field of Condensed Matter Physics. The post will be available from 1 October 1987.

Salary in the Professorial range, current minimum $£$ 19,010 p.a. (under review).

Applications ( 3 copies) to the

Registrar, University of Warwick, Coventry CV4 7AL

from whom further particulars may be obtained (203 523627 ).

Quote Ref. No. 2/A/86 and please mark this clearly on envelope.

Closing date for applications is 7 November 1986. 